\title{
Radiological and bronchoscopic findings for the better yield of tissue diagnosis in lung malignancy
}

\author{
Rabindra Khunjeli ${ }^{1}$, Sanjeet Krishna Shrestha², Manan Karki ${ }^{3}$, Brajendra Srivastava ${ }^{1}$, Ushab Rana \\ Mohsin ${ }^{1}$. \\ ${ }^{1}$ Department of Respiratory Medicine, Shree Birendra Hospital; ${ }^{2}$ National Academy of Medical \\ Sciences, Kathmandu, Nepal; ${ }^{3}$ Research volunteer, Department of Respiratory Medicine, Shree \\ Birendra Hospital.
}

\begin{abstract}
Introduction: In flexible bronchoscopy, absence of endobronchial abnormality blinds the bronchoscopist towards the location of the lung lesion. Guided techniques for bronchoscopy are not available in Nepal. Hence, diagnostic yield is very low. There is need to find the factors to improve yield. This study evaluates the radiological and bronchoscopic findings that can help in histo-pathological yield.
\end{abstract}

Methods: This retrospective study included 557 bronchoscopies done for the evaluation of lung malignancy. Radiological, bronchoscopic and histo-pathological abnormalities were classified and evaluated. The study aimed at finding the radiological and bronchoscopic factors commonly associated with positive yield in histopathology.

Results: Among 557 subjects, 490 had abnormal chest X-ray. Mass lesion was seen in 252(45.24\%), cavity in $19(3.41 \%)$, collapse in $93(16.69 \%)$, consolidation in $81(14.54 \%)$, pleural effusion in $28(5.02 \%)$ and fibrosis in $17(3.05 \%)$ subjects. Total of $314(56.37 \%)$ abnormal bronchoscopies included: endobronchial growth 200(35.9\%), narrowing 66(11.84\%), obstruction $107(19.27 \%)$ and ulcer 16(2.8\%). Histopathology was positive in 198(35.54\%) including 179(32.13\%) lung malignancies. The diagnostic yield for getting positive histopathology for the study population was $35.54 \%$, for mass lesion was $40.47 \%$ and for abnormal bronchoscopy was $54.78 \%$. Significant correlation was identified among mass lesion, endobronchial abnormality and positive histo-pathology. Central mass lesion, collapse and consolidation showed significant association with lung malignancy. Significant association was also identified between histo-pathology and endobronchial growth, obstruction and ulcer.

Conclusions: The diagnostic yield increases with positive endobronchial abnormality. Presence of central lung lesion, collapse and consolidation favour positive yield of histo-pathology. Endobronchial growth, obstruction and ulcer are likely to yield malignant histopathology reports.

Keywords: flexible bronchoscopy;diagnostic yield;lung cancer;epidemiology.

\section{INTRODUCTION}

The flexible bronchoscopy (FB) is the first line of invasive procedure done for the evaluation of lung mass or nodules. A bronchoscopist can take bronchial wash (BW), broncho-alveolar lavage (BAL), protected brush specimen (PBS) or endobronchial lung biopsy (EBLB), under direct visualisation of the lesion. However,

Correspondence:

Dr. Rabindra Khunjeli

Department of Respiratory Medicine, Shree Birendra

Hospital

Email:mrkhunjeli@yahoo.com 
in the abscence of endobronchial abnormalities, bronchoscopist is blinded towards the location of the lesion. ${ }^{1}$

Internationally, guided bronchoscopies are in vogue, which increases the diagnostic yield. This includes: endobronchial ultrasound (EBUS), electromagnetic navigation bronchoscopy (EMNB) and fluoroscopy guided bronchoscopy (FGB). ${ }^{2}$

In Nepal all these modalities are rarely available. The transbronchial needle aspiration (TBNA) is always conducted without guidance; often for large subcarinal lymphnodes. The diagnostic yield of conventional bronchoscopy is less as compared to newer guided techniques. The overall diagnostic yield of bronchoscopy for a given radiological abnormality was around $55.4 \%$ in $1993{ }^{3}$ whereas, the diagnostic yield without guidance is $55.8 \%$ for lung mass or nodule in $2010 .^{1}$

For the conventional techniques without guidance, sampling method also affects the yield. American College of Chest Physician (ACCP) statements on establishing diagnosis of lung cancer states the sensitivity of FB with TBLB to be around $74 \%$ for TBLB, $48 \%$ for BW and $79 \%$ for PBS for central lesions. But for the peripheral lesions (PL) it is 57\%, $43 \%$ and $54 \%$ for EBLB, BAL and PBS respectively even with fluoroscopy guidance. Only with radial EBUS (R-EBUS) the sensitivity can go upto $73 \%$ for PL. ${ }^{4}$ However, overall diagnostic yield with the use of EMNB reaches $80 \%$ for obtaining sample, $74 \%$ for PL and $100 \%$ for lymph nodes. ${ }^{5}$

Since, all the guided bronchoscopic techniques are not available; there is a need for finding factors which can increase diagnostic yield in conventional FB. This study evaluates the radiological and bronchoscopic findings that can affect histo-pathological yield.

\section{METHODS}

This was a retrospective study which included all bronchoscopies conducted at Shree Birendra Hospital (SBH) under Nepalese Army Institute of Health Sciences, a tertiary care centre at Kathmandu. A total of 557 bronchoscopies were included from April 2003 to April 2013. All subjects evaluated for lung nodule, mass or with suspicion of malignancy were included.
All the bronchoscopy registries (BR) were examined and the records with complete data were included. The radiological abnormalities were entered from data registry and confirmed from the radiological library at the department of respiratory medicine. The radiological findings were classified as: normal, abnormal: mass, cavity, collapse, consolidation, pleural effusion and fibrosis. These lesions were further classified according to the location as: central, hilar, peripheral, upper, middle and lower lobe.

All bronchoscopies were conducted under the local protocol of the department. Local anaesthesia with 15\% Lignocain spray and 2\% lignocaine solution under direct vision were routinely used. FB was inserted through nasal route. Larynx, trachea and bronchial tree were inspected till the sub-segmental level. The normal side, as per radiology was inspected first. Any abnormality in the tracheobronchial tree was noted from the records. The abnormalities were classified as: endobronchial growth (EBG), endobronchial narrowing (EBN), Endobronchial obstruction (EBO), Endobronchial Ulceration (EBU), Extra-luminal compression (ELC) and anatomical distortion (AD). FBs were conducted by faculties of respiratory medicine and senior residents. The number and type of sampling done during the procedure was at the discretion of the bronchoscopist which included: BW, BAL, PBS, EBLB and TBNA. All the samples obtained were subjected to cytological and histopathological examination (HPE) as per protocol. Reports were noted down from BR and traced from the department of pathology and microbiology. Subjects without positive HPE were subjected to ultrasound or Computed tomography (CT) guided fine needle aspiration cytology (FNAC). HPE reports were classified as: Normal and Abnormal. The abnormal reports included: Small cell lung cancer (SCLC), Nonsmall cell lung cancer (NSCLC), undifferentiated carcinoma and others included carcinoid. NSCLC was further stratified as: Squamous cell carcinoma (SQC), Adenocarcinoma (ADC), Large cell carcinoma (LCC) and undifferentiated NSCLC.

Descriptive statistics was used for initial evaluation of demographical characterization of collected data. Categorical data were expressed as absolute counts and percentage. The numerical data were expressed as mean \pm standard deviation (SD). At $95 \%$ confidence statistical significance was set at $\mathrm{p}<0.05$. Chi square test and Fischer's exact test were used for categorical data. 


\section{RESULTS}

A total of 557 entries qualified for the analysis with complete records from April 2003 to April 2013. The maximum referrals to respiratory medicine were for abnormal chest $\mathrm{X}$ ray (CXR). The mean age of study population was $59.69 \pm 14.43$ years. $71.8 \%$ of the study group were males with male female ratio of 2.56:1.
Among 557 subjects, 67 had normal CXR. Mass lesion was seen in 252(45.24\%) subjects, cavity was seen in $19(3.41 \%)$, collapse in $93(16.69 \%)$, consolidation in $81(14.54 \%)$, pleural effusion in $28(5.02 \%)$ and fibrosis in 17(3.05\%). Among 557 bronchoscopies, $314(56.37 \%)$ were abnormal. The most common finding was EBG in 200(35.9\%), EBN 66(11.84\%), EBO 107(19.27\%) and EBU 16(2.8\%) subjects. The common radiological and endobronchial abnormalities are enlisted in table 1.

Table 1.Radiological and Bronchoscopic abnormalities in the study population

\begin{tabular}{|l|l|l|c|}
\hline Radiological Findings & $\mathrm{N}($ percent of total) & Bronchoscopic Findings & $\mathrm{N}($ percent of total) \\
\hline Normal & $67(12.03 \%)$ & Normal & $238(42.72 \%)$ \\
\hline Abnormal & $490(87.97 \%)$ & Abnormal & $314(56.36 \%)$ \\
\hline Total & $557(100 \%)$ & Abandoned & $5(0.92 \%)$ \\
\hline & & Total & $557(100 \%)$ \\
\hline & & Endobronchial Growth & $200(35.90 \%)$ \\
Mass & $252(45.24 \%)$ & Endobronchial Narrowing & $66(11.84 \%)$ \\
Cavity & $19(3.41 \%)$ & Endobronchial Obstruction & $107(19.27 \%)$ \\
Collapse & $93(16.69 \%)$ & Endobronchial Ulcer & $16(2.8 \%)$ \\
Consolidation & $81(14.54 \%)$ & Extra Luminal Compression & $23(4.12 \%)$ \\
Pleural Effusion & $28(5.02 \%)$ & Anatomical distortion & $31(5.56 \%)$ \\
Fibrosis & $17(3.05 \%)$ & Copious Secretions & $2(0.36 \%)$ \\
\hline
\end{tabular}

Note: More than one finding in a single subject was entered separately.

When positive HPE and cytology reports were evaluated, total 198(35.54\%) reports were identified. Total 179(32.13\%) samples were positive for lung malignancy. 19(3.41\%) of the samples were labelled as benign. The histological characteristics of the study population are enlisted in table 2 .

For our statistically evaluation we included 252 subjects with mass or nodule in CXR or CT chest. In this group only $168(66.67 \%)$ had positive findings in FB and 102 (40.47\%) had positive histopathology. Hence, the overall diagnostic yield for getting HPE positive sample from FB without guidance in radiological mass or nodular lesion was around $40.47 \%$ in our setup. However, the overall yield for getting HPE positive (both benign and malignant) report from any radiological abnormality was only $35.54 \%$. When subjects with bronchoscopic abnormalities wereconsidered the diagnostic yield increased to $54.78 \%$.
Table 2. Histological distribution of the study population

\begin{tabular}{|c|c|c|}
\hline Benign & & 19 \\
\hline $\begin{array}{l}\text { Aspergillosis } \\
\text { Tuberculosis } \\
\text { Sarcoidosis } \\
\text { Vascular Ectasia } \\
\text { Hemangioma } \\
\text { Benign Polyp } \\
\text { Leiomyoma }\end{array}$ & $\begin{array}{l}3 \\
6 \\
3 \\
1 \\
2 \\
1 \\
3\end{array}$ & \\
\hline Malignant & & 179 \\
\hline $\begin{array}{l}\text { Small cell carcinoma } \\
\text { Non-Small Cell Carcinoma } \\
\text { Squamous Cell Carcinoma } \\
\text { Adenocarcinoma } \\
\text { Large Cell Carcinoma } \\
\text { Undiferentiated NSCLC } \\
\text { Undifferentiated Carcinoma } \\
\text { Carcinoid }\end{array}$ & $\begin{array}{l}32 \\
141 \\
113 \\
22 \\
1 \\
4 \\
6 \\
1 \\
\end{array}$ & \\
\hline Total & & 198 \\
\hline
\end{tabular}


When cross tabulation was done between radiological mass lesion and presence of positive endobronchial abnormality, significant positive correlation was identified with $\mathrm{p}<0.05$. Similarly, significant positive correlation was seen between radiological mass lesion and positive HPE reports.

As a second part of our evaluation we tried to identify associations between various radiological findings with 179 malignant HPE. The most common radiological side of occurrence of lung lesion was on the right side $(88$ subjects $=49.16 \%)$. There were $82(42.18 \%)$ cases with left sided lesion and only $9(5.01 \%)$ had bilateral lesion. However, no statistically significant difference was seen between all three categories as compared with HPE.

Upper lobe lesions were predominant amounting to $62(34.63 \%)$, followed by $39(21.87 \%)$ lower lobe lesions and 38(19.55\%) middle lobe lesions. $40(22.34 \%)$ patients had hilar pathology and $30(16.75 \%)$ had para-tracheal pathology. However, statistically association was identified between HPE and lower lobe lesions only $(\mathrm{p}=0.013)$.

Among 179 subjects with positive HPE, 102(56.98\%) presented with radiological mass or nodular lesions, 64(35.75\%) with collapse and 31(17.31\%) with consolidation. The least common features were pleural effusion $(5.02 \%)$, cavity $(2.79 \%)$ and fibrosis $(2.23 \%)$. In 557 patients, the radiological findings which showed positive associations with positive HPE were presence of central mass lesion $(p=0.001)$, collapse $(p=0.004)$ and consolidation $(p=0.037)$. However, there was no statistically significant association between positive HPE and cavity $(p=0.657)$, pleural effusion $(p=0.376)$ and fibrosis $(\mathrm{p}=0.268)$.

We further evaluated association of FB findings with histopathology. The statistically significant association was identified between HPE and EBG ( $p=0.001), E B O$ $(p=0.001)$ and $\operatorname{EBU}(p=0.09)$. There was no association with $\operatorname{EBN}(p=0.698)$ and $\operatorname{ELC}(p=0.06)$.

\section{DISCUSSION}

Lung cancer is the leading cause of cancer related deaths in American population. ${ }^{6}$ This stands true for both the genders. In United States 201,144 cases of lung cancer were diagnosed in 2010 which included $53.28 \%$ males and $46.72 \%$ females. $^{7}$ Estimated new cases and deaths from lung cancer (NSCLC and SCLC) in the United States in 2014 are 224,210 and 159,260, respectively. ${ }^{8}$ South East Asian Nations also show similar trend. Lung cancer is the most common cancer in the major cities of India like: Calcutta, Mumbai, and New Delhi. ${ }^{9}$ In the data published in April 2011 lung cancers deaths in Nepal reached 3,100 or 2.09\% of total deaths. The age adjusted death rate is $19.36 \mathrm{per}$ 100,000 of population. According to this data Nepal is in $68^{\text {th }}$ rank for lung cancer related deaths. ${ }^{10}$

The burden of lung malignancy is global. It was in its peak in 1980s and even started to plateau for females in United States. ${ }^{6}$ But the countries like Nepal are still struggling with the basic diagnostic modalities. Video bronchoscope without guide is the modality used frequently. In this study we retrospectively evaluated our bronchoscopy registry. Among the 557 complete entries, $45.60 \%$ were referred for lung mass/nodule. Our average age for the referral for suspected lung malignancy was $59.64 \pm 14.43$ years, which is in accord with age group for lung cancer. Significantly higher numbers of males were referred; this might have been due to Armed Force setup.

In this study overall bronchoscopic yield for positive HPE was only $33.54 \%$. When same evaluation was done for only mass/nodules, our yield increased to $40.71 \%$. These values are very low as compared to $55.8 \%$ diagnostic yield of conventional bronchoscopy. ${ }^{1}$ However, consideration of only visible abnormalities in FB, the diagnostic yield increased to $54.78 \%$. These values were significantly low as compared to $80 \%$ overall diagnostic yield of $\mathrm{EMNB}^{5}$ and $71 \%$ for EBUS TBNA. ${ }^{11}$

This study also evaluated the prevalence of major histological subtypes of epithelial lung cancers. The major histological subtypes were: squamous cell carcinoma $(63.13 \%)$ and small cell carcinoma (17.88\%). Traditionally, these are the two major subtypes associated with heavy smoking. ${ }^{6}$ Our study also showed statistically significant association between smoking and these histological subtypes. On the contrary adenocarcinoma was the third most common subtype amounting to $12.29 \%$. This was in striking contrast with the international trend, where adenocarcinoma is the predominant lung cancer subtype. ${ }^{6}$

In a study conducted in Japan on Osaka Cancer Registry spanning over 33 years, there was a gradual increase in lung cancer incidence with shift of 
predominant histological subtypes from SQC to ADC. The predominant change occurred in 1990s according to the data. ${ }^{12}$ In our study the SQC and SCLC remained the predominant histological subtypes every year from 2003 to 2013. There was a statistically significant association between all diagnosed cases and smoking. There were $67.35 \%$ of smokers in the referred population. Hence, the smoking associated histological subtypes. The predominance of SQC and SCLC in our study may be attributed to the smoking behaviour of Nepali men and women.

In journal published in 2010, SQC was found to be the most common histological type $(n=312,67.5 \%)$ in Kashmir valley in India followed by SCLC(n = $96,20.8 \%)$ of the cases. There were $14(3 \%)$ cases of ADC. ${ }^{13}$ In the study conducted in Chandigarh, 1009 cases of lung cancers, Jindal and Behera reported that $34.3 \%$ of the patients had SQC, $27.8 \%$ had SCLC, $25.9 \%$ had ADC and $12.2 \%$ were unclassified variety. ${ }^{14}$ In the analysis of lung cancer in Karachi Cancer Registry in 2006, the proportion of SQC, SCLC, ADC and LCC were $61 \%, 12.5 \%, 6.9 \%$ and $11.6 \%$ in males from 1995 to 1997 . The proportion changed to $34.8 \%$, $16.2 \%, 10.7 \%$ and $14 \%$ in between 1998 to 2002 , respectively. In this study there was a notable increase in incidence of ADC in males. However, there was no significant change of ADC in females. ${ }^{15}$ Our study also showed the histological distribution of lung cancer in accord to South East Asian Nations, but did not show the increase in ADC.

In Nepal pulmonologist are always performing video bronchoscopy without guidance. The recent guided techniques are not available in most of the centres. With this view this study tried to identify the common factors in the radiology and bronchoscopy which will help in positive yield of histopathology for diagnosis.

This evaluation showed that right sided and upper lobe lesions were more frequent without statistical significance. In a Turkish study published in Asia Pacific Journal of Cancer Prevention in 2011, right sided lesion was the most common side for lung malignancy. The upper lobes were the commonest distribution. ${ }^{16}$ Our study did not find any significance relation between upper and middle lobe lesions with positive yield of HPE. However, there was significance association with lower lobe lesions. Our study also showed that central mass lesion was the most common abnormality associated with lung malignancy. The central mass lesion, collapse and consolidation had statistically significant chance for the yield of positive HPE. Similarly, EBG, EBO and EBU had highest yield for positive HPE with significant statistical association.

In 1993, Wei-Juin Su et al. published CXR guidelines in the selection of patients for FB. In this study mass lesion $(36.2 \%)$ in radiology was statistically associated with lung cancer. Mass lesion (47\%) and lobar collapse $(64.8 \%)$ were the most common radiological findings associated with positive bronchoscopy. Our study also showed positive correlation between mass lesion and positive bronchoscopy. ${ }^{3}$

In a more detailed evaluation for the factors affecting the yield of bronchoscopy done in 2010, the univariate analyses revealed cough, tumour size, endobronchial visibility and characteristics of endobronchial abnormalities as the positive predictors of diagnostic yield. In the same study multivariate analysis showed independent association with tumour size. In our study we did not evaluate the tumour size and its correlation with HPE yield. The retrospective nature prevented from actual sizing of the lesion. One similarity of our study to this study was the increase in diagnostic yield with a visible FB abnormality (from $40.71 \%$ to $54.78 \%)^{1}$

\section{CONCLUSIONS}

This study showed that radiology is an important initial evaluation for lung malignancy. The central lung lesion, collapse and consolidation were more commonly associated with diagnosed lung malignancy. EBG, EBO and EBU were FB findings more likely to yield malignant HPE reports. Endobronchial abnormality in FB increases the diagnostic yield of FB without guidance. These radiological and bronchoscopic factors are associated with significant chances of yielding malignant HPE and should be taken into consideration during evaluation of the patient. Lastly, our study revealed that SQC, SCLC and ADC, in the descending order, are the most common HPE types in Nepal. 


\section{REFERENCES}

1. Boonsarngsuk V, Raweelert P, Sukprapruet A, Chaiprasithikul R, Kiatboonsri S. Factors affecting the diagnostic yield of flexible bronchoscopy without guidance in pulmonary nodules or masses. Singapore Med J. 2010 Aug;51(8):660-5.

2. Fuhlbrigge AL, Choi AMK. Diagnostic procedures in Respiratory Disease. In: Dan LL, Kasper DL, Jameson JL, Fauci AS, Hauser SL, Loscalzo J, editor. Harrison's principles of Internal Medicine. 18th ed. New York (NY): McGraw-Hill; 2012. p. 2099-100.

3. Su WJ, Lee PY, Perng RP. Chest roentgenographic guidelines in the selection of patients for fiberoptic bronchoscopy.Chest. 1993;103(4):1198-201.http:// dx.doi.org/10.1378/chest.103.4.1198

4. Detterbeck FC, Lewis SZ, Diekemper R, AddrizzoHarris DJ, Alberts WM. Diagnosis and Management of Lung Cancer, 3rd ed: American College of Chest Physicians Evidence-Based Clinical Practice Guidelines. Chest 2013;143(5) Suppl:S7-37.

5. Gildea TR, Mazzone PJ, Karnak D, Meziane M, Mehta AC. Electromagnetic Navigation Diagnostic Bronchoscopy: A Prospective Study. Am J Respir Crit Care Med. 2006 Nov;174(9):982-9. http://dx.doi. org/10.1164/rccm.200603-3440C.

6. Horn L, Pao W, Johnson DH. Neoplasms of the lung. In: Longo DL, Fauci AS, Kasper DL, Hauser SL, Jameson JL, Loscalzo J, editors. Harrison's Principles of Internal Medicine. 18th ed. New York: McGraw-Hill; 2012. p.737-8.

7. Centers for Disease Control and Prevention. CDC - Lung cancer statistics [Internet]. Georgia. 2014 September 2 [8 September 2014]. Available from: http://www.cdc. gov/cancer/lung/statistics/

8. National Cancer Institute. Lung Cancer [Internet]. 2014 [8 September 2014]. Available from: http://www.cancer. gov/cancertopics/types/lung/

9. Mudur G. India has some of the highest cancer rates in the world. BMJ. Jan 29, 2005;330(7485):215.http:// dx.doi.org/10.1136/bmj.330.7485.215-c

10. World Life Expectancy. Lung Cancers in Nepal. [Internet]. 2014 [8 September 2014]. Available from: http://www.worldlifeexpectancy.com/nepal-lungcancers

11. Karunamurthy A, Cai G, Dacic S, Khalbuss WE, Pantanowitz L, Monaco SE. Evaluation of endobronchial ultrasound-guided fine-needle aspirations (EBUSFNA): correlation with adequacy and histologic followup. Cancer Cytopathol. 2014 Jan;122(1):23-32.http:// dx.doi.org/10.1002/cncy. 21350

12. Toyoda Y, Nakayama T, Ioka A, Tsukuma H. Trends in lung cancer incidence by histological type in Osaka, Japan. Jpn J ClinOncol. 2008 Aug;38(8):534-9.http:// dx.doi.org/10.1093/jico/hyn072

13. Koul PA, Kaul SK, Sheikh MM, Tasleem RA, Shah A. Lung cancer in the Kashmir valley. Lung India. 2010 Jul-Sep;27(3):131-7.http://dx.doi.org/10.4103/09702113.68309

14. Jindal SK, Behera D. Clinical spectrum of primary lung cancer - review of Chandigarh experience of 10 years. Lung India. 1990;8:94-8.

15. Bhurgri Y, Bhurgri A, Usman A, Sheikh N, Faridi N, Malik J, et al. Patho-epidemiology of lung cancer in Karachi (1995-2002). Pac J Cancer Prev. 2006 JanMar;7(1):60-4.

16. Sahin F, Yildiz P. Radiological, Bronchoscopic and Histopathologic Characteristics of Patients with Primary Lung Cancer in Turkey (2006-2009). Asian Pacific J Cancer Prev. 2011;12(8):1947-52. 